\title{
Susceptibilidad a inundación por flujos torrenciales a partir de la integración del método geomorfológico y de la modelización con FLO-2D, lago Atitlán, Guatemala
}

\section{Sergi Paricio Salas ${ }^{1, *}$, Marta Guinau Sellés ${ }^{2}$, Joan Bach i Plaza ${ }^{3}$, y Luis Iván Girón Melgar ${ }^{1}$}

${ }^{1}$ Área de Gestión Ambiental y de Riesgos, Asociación Vivamos Mejor; Calle de los Salpores, 0-83, Zona 3, Barrio Jucanyá, Panajachel, Departamento de Sololá, Guatemala.

${ }^{2}$ Grup de Recerca RISKNAT, Departament de Dinàmica de la Terra i de l'Oceà, Universitat de Barcelona, C/ Martí i Franquès s/n, 08028 Barcelona, Catalunya, España.

${ }^{3}$ Unitat de Geodinàmica Externa i d'Hidrogeologia, Departament de Geologia, Facultat de Ciències, Universitat Autònoma de Barcelona, Edifici Cs, 08193 Cerdanyola del Vallès, Catalunya, España.

*pariciosergi@gmail.com

\section{RESUMEN}

El lago Atitlán (Guatemala) se encuentra localizado en la zona de paso de los ciclones tropicales formados tanto en el océano Atlántico como en el Pacífico. Este tipo de fenómenos provocan anualmente abundantes e intensas precipitaciones que, a su vez, generan numerosas inundaciones y la formación de flujos torrenciales. El presente artículo pretende profundizar en el conocimiento de este tipo de procesos y en la predicción de su alcance espacial. El trabajo se ha desarrollado en la cuenca de Tzununá y se han tomado como referencia el huracán Stan (2005) y la tormenta tropical Agatha (2010). Durante su paso por la región se registraron máximas de precipitación en dos días de 383 y $269 \mathrm{~mm}$, respectivamente. Para el análisis se ha aplicado el método geomorfológico y se han modelizado flujos con diferentes propiedades reológicas a partir del software FLO-2D. El trabajo de reconocimiento geomorfológico de detalle ha sido esencial y ha permitido validar algunos de los resultados de las modelizaciones y descartar, o dar menor peso, a otros menos representativos de la realidad. La integración de los resultados de ambas metodologías ha permitido la elaboración de un mapa de susceptibilidad a inundación más detallado y de mayor validez.

Palabras clave: geomorfología; susceptibilidad a inundación; flujos torrenciales; FLO-2D.

\section{ABSTRACT}

Lake Atitlan (Guatemala) is located in the transit region of the tropical cyclones formed in both the Atlantic Ocean and the Pacific Ocean. Every year, these phenomena cause abundant and intensive rain fall which in turn provokes floods associated with the formation of destructive flows. This paper aims to deepen the knowledge of such processes and the prediction of their spatial distribution. The work was carried out in the Tzununá basin, taking as reference phenomena Hurricane Stan (2005) and Tropical Storm Agatha (2010). When they passed through the region, maximum rain falls of 383 and $269 \mathrm{~mm}$ were registered on two consecutive days. In this work, the geomorphological method was developed and flows were simulated with different rheological properties using FLO-2D software. The geomorphological detailed exploration field work has been essential and has allowed the validation of some of the model results. It has also enabled us to discard others wich are not representative of real conditions. The integration of the results of both methodologies has made possible the creation of a more detailed and validated inundation susceptibility map.

Key words: geomorphology; inundation susceptibility; torrential Flow; FLO-2D.

\section{INTRODUCCIÓN}

El territorio que abarca Centroamérica y el centro y sur de México sufre anualmente graves inundaciones y numerosos procesos de inestabilidad de ladera como consecuencia del paso de ciclones tropicales formados durante la época de lluvias (entre mayo y octubre). Según el National Hurrican Center (NHC, 2018), solo en el año 2010, la región se vio afectada por siete ciclones tropicales, cuatro de ellos llegaron a la categoría de huracán y el resto a la de tormenta tropical. En Guatemala, el paso de la tormenta tropical Agatha ese mismo año provocó la pérdida de 160 vidas humanas y unos daños estructurales valorados en 982 millones de dólares (Beven, 2010). Para mitigar los efectos asociados a la ocurrencia de eventos hidrometeorológicos extremos, algunos autores como Montoya-Jaramillo et al. (2009) y Sepúlveda et al. (2016) proponen un análisis multidisciplinario de la peligrosidad asociada a estos fenómenos, con el objetivo de generar una planificación urbanística adecuada (Hürlimann et al., 2006).

El presente trabajo tiene el objetivo de evaluar la susceptibilidad a inundación por flujos torrenciales de la cuenca de Tzununá (Departamento de Sololá, Guatemala), tributaria del lago Atitlán y localizada en su margen norte (Figura 1). Debido a los escasos recursos económicos de la región, el desarrollo sociourbanístico de los diferentes municipios y comunidades ubicados a las orillas del lago Atitlán (Figura 1b) 
se ha dado, en su mayor parte, sobre superficies correspondientes a abanicos aluviales de extensión inferior a $2.5 \mathrm{~km}^{2}$. Estos se caracterizan por presentar una gran actividad hidrológica durante la época de lluvias, lo que hace que dichas zonas queden sujetas a un alto grado de riesgo de afectación por flujos torrenciales. Los tres municipios más poblados que bordean el lago Atitlán, Santiago de Atitlán (49631 hab.), San Lucas Tolimán (32480 hab.) y Panajachel (19157 hab.) (Instituto Nacional de Estadística de Guatemala, 2015) se encuentran localizados sobre zonas de abanico aluvial y se vieron afectados, tanto en el año 2005 como en el 2010, por los efectos destructivos derivados del huracán Stan y de la tormenta tropical Agatha respectivamente.

La presente investigación se basa en la aplicación de una metodología multidisciplinaria, con la integración del método geomorfológico y el modelado numérico de los procesos observados utilizando el código FLO-2D. Para ello, se han tomado como referencia las precipitaciones registradas durante la tormenta tropical Agatha en el 2010 y los efectos provocados por dicha tormenta y por el huracán Stan, en el 2005, en la zona baja de la cuenca de Tzununá (lago Atitlán - Guatemala) (Figura 1). Adicionalmente, se presenta una validación de la metodología utilizada y de los resultados obtenidos mediante un proceso de filtrado y contraste de los datos cualitativos y cuantitativos obtenidos. Esto ha permitido ajustar los modelos desarrollados y definir con mayor precisión los diferentes niveles de susceptibilidad.

\section{TERMINOLOGÍA Y ANTECEDENTES}

Dada la gran diversidad de términos y clasificaciones de los fenómenos que se tratan en este estudio, es necesario definir la terminología utilizada. De este modo, se ha decidido utilizar el término "flujo torrencial" para designar cualquier tipo de flujo de carácter extraordinario. Según la comparativa entre las cantidades de lluvia precipitada durante los fenómenos hidrometeorológicos de relevancia ocurridos desde 1998 (Figura 2) y los flujos generados, se considera flujo extraordinario a aquel generado por precipitaciones acumuladas en un día superiores a los $125 \mathrm{~mm}$ o por precipitaciones acumuladas entre dos y cuatro días superiores a los $250 \mathrm{~mm}$ (Paricio-Salas, 2011). De la misma manera se considera flujo ordinario al flujo generado por precipitaciones cuyas cantidades acumuladas no superan los umbrales anteriores. La tipología y características de los diferentes tipos de flujos torrenciales varía en función del porcentaje de carga sólida que transportan y de sus características reológicas. Estos pueden ser: a) flujos acuosos o stream flows ( $<20 \%$ en volumen de carga sólida), b) flujos hiperconcentrados o hyperconcentrated flows (entre un 20 y un $60 \%$ en volumen de carga sólida) o c) flujos de detritos o debris flows (>60\% en volumen de carga sólida) (Beverage y Culberston, 1964; Pierson y Costa, 1987). Se considera como "inundación repentina" (flash flood) la inundación consecuencia de un flujo torrencial generado por precipitaciones de

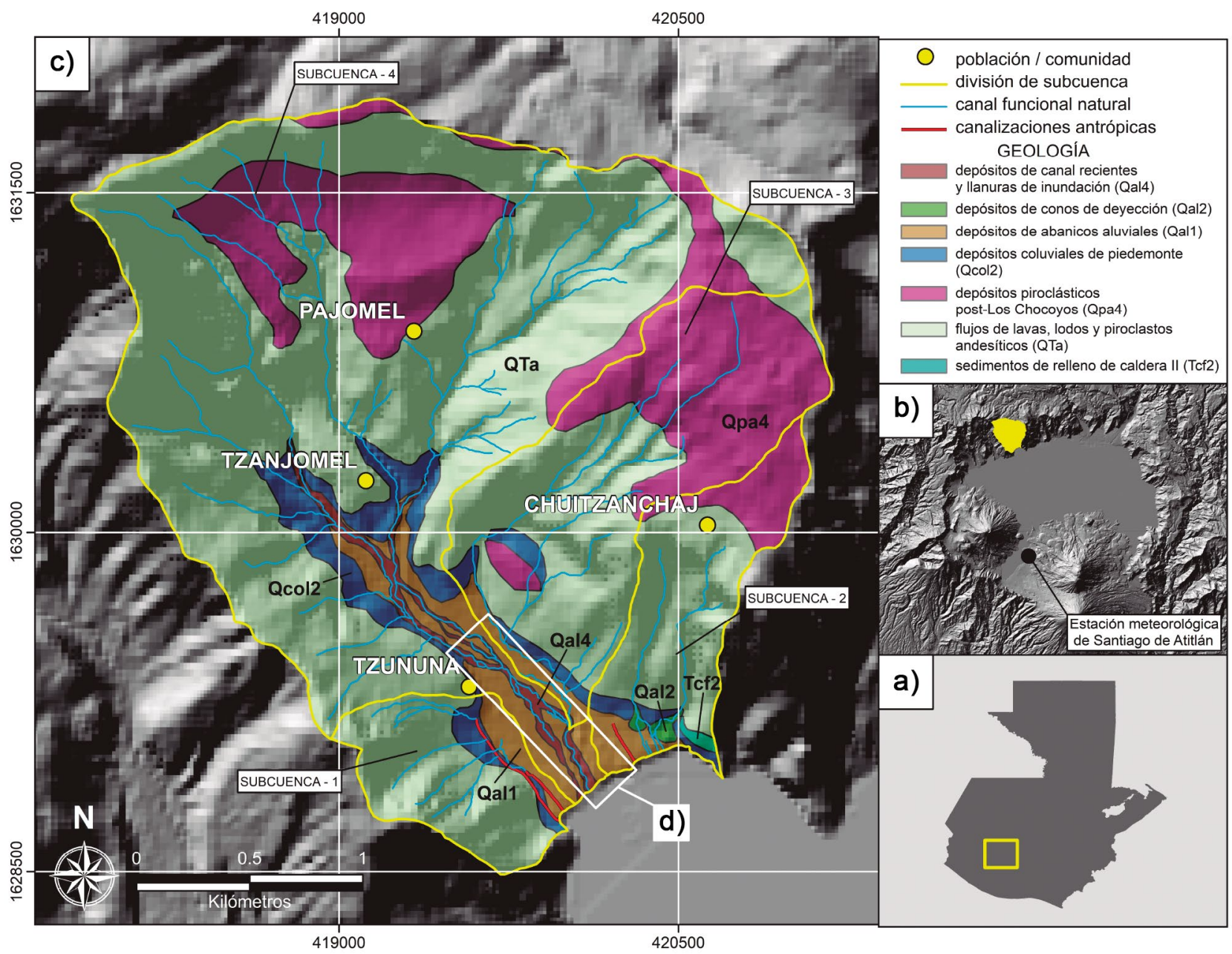

Figura 1.a) Localización de la región del lago Atitlán (recuadro amarillo) en Guatemala (polígono gris oscuro). b) Localización de la cuenca de Tzununá en el lago Atitlán. c) Red hidrológica, división en subcuencas y geología de la cuenca de Tzununá. d) Tramo bajo del canal principal (ver ampliación en Figura 5). Sistema geográfico de referencia: GTM (Guatemala Transversa Mercator) / Datum: WGS 1984. Fuente de la cartografía geológica: Núñez-Álvarez y Martínez-Cobo, 2011b. 


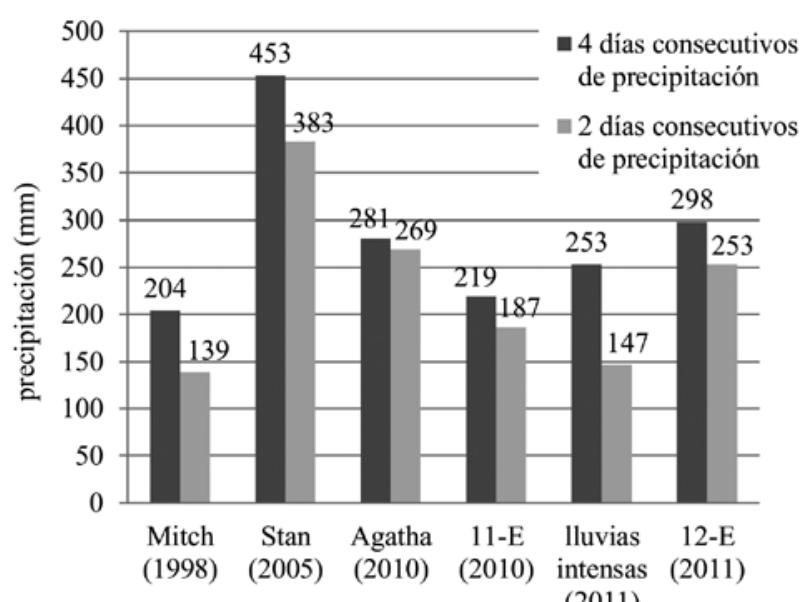

(2011)

Figura 2. Valores de precipitación acumulada entre los dos y los cuatro días de máxima precipitación de los fenómenos meteorológicos más relevantes acontecidos entre 1998 y 2013. Fuente: INSIVUMEH, 2013, Instituto Nacional de Sismología, Vulcanología, Meteorología e Hidrología.

alta intensidad. Y finalmente, se usa el término "movimiento de ladera" haciendo referencia a cualquier tipo de desplazamiento de una masa de material ladera abajo.

Los flujos acuosos, aún y pudiendo transportar un cierto porcentaje de carga sólida, ya sea en suspensión o como carga de fondo, conservan las características de un flujo de comportamiento newtoniano (Pierson, 2005). Los flujos hiperconcentrados son bifásicos, heterogéneos, no newtonianos, de tipo turbulento, no cohesivos y con tensiones dispersivas dominantes (Brea et al., 2005). Los flujos de detritos también presentan un alto grado de heterogeneidad, son no newtonianos, laminares, con un gran potencial de incorporación de material durante su transporte, un caudal máximo que supera fuertemente caudales puramente acuosos y la presencia de diferentes pulsaciones o frentes durante un mismo evento (Bateman et al., 2007). Sus propiedades reológicas dependen de diversos factores que controlan el tipo de interacción entre granos, como la concentración de la fracción de sólidos, el tamaño y la forma de grano y el porcentaje de partículas finas (limo y arcilla) que pueden incrementar la cohesión del flujo (Lin et al., 2011).

\section{Procesos de remoción en masa y formación de flujos torrenciales}

Durante y tras periodos de precipitaciones prolongadas o intensas, las partes medias y altas de las laderas de la cuenca se ven densamente afectadas por multitud de movimientos de ladera y flujos. Según VanDine (1996), el inicio de estos fenómenos requiere de una pendiente mayor a los $25^{\circ}(47 \%)$. Vilaplana et al. (2002) recomiendan que en contextos geológicos similares al de la zona de estudio, y partiendo de datos topográficos de escasa calidad y baja precisión, se establezca un factor de seguridad y se reduzca dicho ángulo a $22^{\circ}$. Así pues, las características de los materiales afectados por movimientos de ladera, las elevadas pendientes de la zona y el grado de saturación del suelo conforman los factores condicionantes que controlan este tipo de procesos.

La probabilidad de que se generen flujos torrenciales y de que contengan o no un cierto porcentaje de carga sólida depende esencialmente de tres factores:

1. De la precipitación acumulada durante el episodio de lluvias extraordinario. Es importante destacar que la intensidad de la precipitación es un factor determinante y puede dar lugar, en función de las dimensiones y de la morfología de la cuenca, a fenómenos de tipo inundación repentina, incluso cuando las cantidades acumuladas no alcancen los valores anteriormente establecidos.

2. De las condiciones de humedad del suelo anteriores a un episodio de lluvias extraordinario. En ese sentido, el periodo crítico de condiciones climáticas previas influyentes en la saturación del suelo puede ser muy variable y depende de la tipología de los suelos, de la cantidad de precipitación antecedente y del clima. Capra et al. (2010) definen tres días como el periodo de tiempo crítico, otros autores como Pasuto et al. (1998) establecen periodos superiores de hasta 15 días.

3. De la cantidad de material disponible a lo largo de la extensión que cubre la zona de inicio y trayecto del flujo. Así pues, la probabilidad de que se den flujos con carga sólida disminuye los años posteriores a un evento de gran magnitud ya que las canalizaciones quedan limpias y desprovistas de material potencialmente disponible para ser movilizado e incorporado al flujo. Este aspecto se aprecia con claridad si comparamos la diferencia entre el número de movimientos de ladera y flujos torrenciales que se dieron para Stan (2005) y para Agatha (2010).

De esta manera, el aumento del porcentaje de material aportado al flujo principal por movimientos de ladera y flujos secundarios genera un incremento de la carga sólida que hace que estos procesos, inicialmente acuosos, puedan evolucionar en un mismo evento a flujos hiperconcentrados y, si se dan las condiciones necesarias, transformarse en flujos de detritos. Debido a la alta intensidad de las precipitaciones y al escaso tiempo de concentración de este tipo de cuencas, los procesos descritos pueden llegar a darse de forma rápida y efímera y dar lugar a fenómenos de tipo inundación repentina.

El desbordamiento de los flujos generados siempre se produce sobre las zonas de abanico aluvial. Esto puede deberse a que la capacidad portante del cauce principal no es suficiente para canalizar el volumen de agua y material transportado, a que los márgenes de la canalización, en caso de que existan, se desestabilizan y acaban rompiéndose o a una combinación de ambos factores. Estos márgenes pueden ser naturales, generados por la propia dinámica incisiva del río, o antrópicos. Los márgenes antrópicos construidos en la zona suelen ser de dos tipos: a) muros de gaviones o b) muros de material dragado del lecho del propio río. Los muros de gaviones suelen ser efectivos para pequeñas avenidas, pero poco eficaces para soportar el empuje de flujos torrenciales puesto que, debido a la erosión basal, se desestabilizan y acaban volcando (Figura 3a y 3b). Los muros de material dragado (Figura 3c) no son recomendables puesto que dicho material no se suele consolidar $y$, por lo tanto, queda disponible para ser erosionado e incorporado al flujo, aunque este no sea de carácter extraordinario.

\section{Estudio de los flujos torrenciales}

Existen diferentes metodologías que permiten analizar las características de este tipo de fenómenos y su distribución y alcance espacial. El método geomorfológico integrado, desarrollado por algunos autores como Fernández-Lavado (2010), Serra-Creixell (2011) y Gurdiel-Pérez (2014), es un método cualitativo basado en el análisis histórico del material gráfico disponible y en el trabajo de campo en busca de indicadores geomorfológicos que aporten datos acerca del alcance de fenómenos pasados y de la probabilidad relativa de alcance de fenómenos futuros. Dado que los mapas de inventarios de procesos geodinámicos son una herramienta básica para evaluar los riesgos en una determinada región (Insuasty-Enríquez, 2012), este tipo de análisis y la posterior integración de los datos recogidos en un mapa de indicadores geomorfológicos son esenciales para conocer in situ los fenómenos estudiados. Existen también otras metodologías como el modelo adoptado por la Soil and Water Conservation Bureau of Taiwan, basado en ecuaciones empíricas, que establece la extensión deposicional de los flujos en función del volumen de material implicado y del ángulo de la pendiente por debajo del ápice del abanico aluvial (Hsu et 

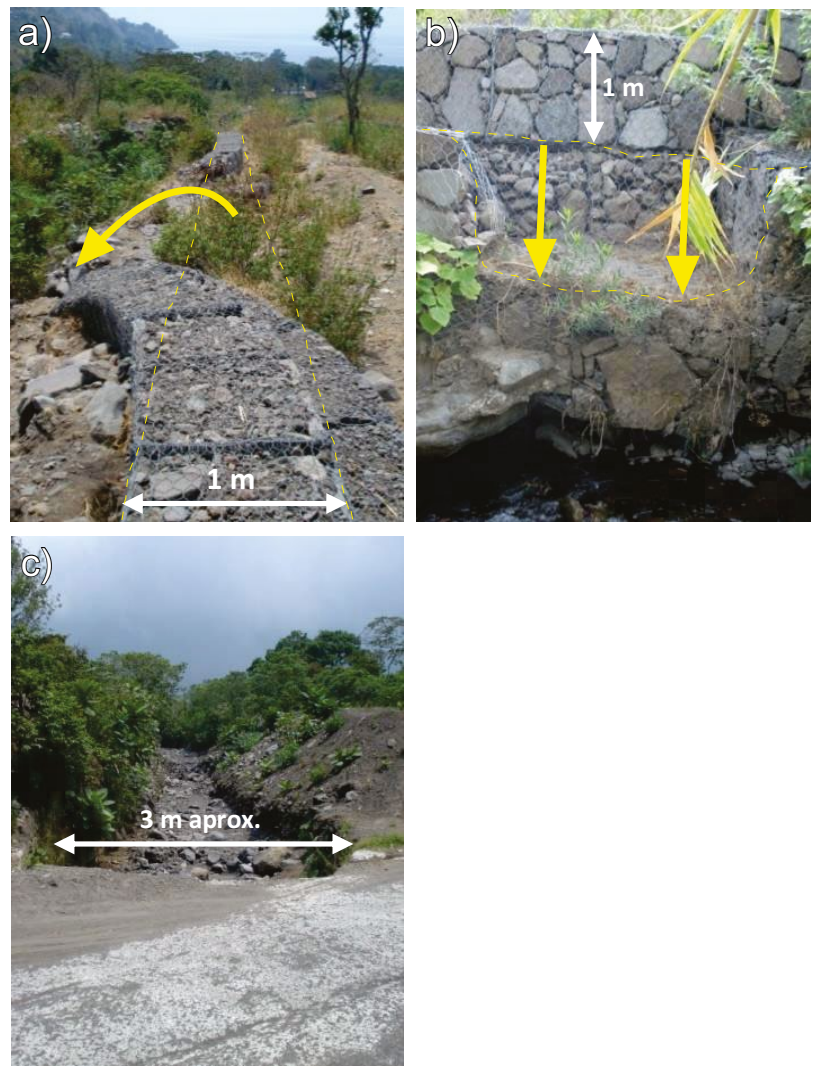

Figura 3. a) Gaviones volcados en el margen derecho de la canalización principal del río. La flecha amarilla indica la dirección del movimiento de los gaviones. b) Muro de gaviones afectado por erosión basal. Las flechas amarillas indican la dirección del movimiento de los gaviones. c) Tramo de canal dragado cercano a la población de Santiago de Atitlán. En la fotografía se observa cómo el material dragado se acumula en los bordes del canal a modo de dique sin realizar sobre él ningún tipo de consolidación.

al., 2010). Finalmente, las líneas de trabajo más recientes, analizan el alcance de este tipo de fenómenos a partir de modelos numéricos que simulan el comportamiento espacial de flujos, tanto acuosos como con carga sólida, en función de diferentes parámetros. Algunos de estos como HEC-RAS, desarrollado por el US Army Corps of Engineers (USACE, 2018), son modelos hidráulicos que permiten simular flujos esencialmente acuosos mediante la generación de perfiles de superficies de agua en regímenes de flujo mixto, subcrítico y supercrítico (Hernández et al., 2012). Otros autores como Oliva-Hernández (2007), Hsu et al. (2010), Lin et al. (2011), Caballero y Capra (2014) y Sepúlveda et al. (2016) han generado modelos a partir del software FLO-2D. Este permite añadir la componente de la carga sólida al flujo a partir de diferentes opciones y, de esta manera, obtener resultados del alcance y la distribución espacial de flujos hiperconcentrados y flujos de detritos. Bateman et al. (2007) desarrollan también un modelo bidimensional para la simulación de flujos llamado FLAT Model, el cual se alimenta de varias mallas de información como la topografía, las condiciones iniciales, las condiciones de contorno, la rugosidad, la profundidad máxima erosionable, etc. Este tipo de programas son una herramienta útil para predecir, de manera aproximada, el comportamiento hidráulico de los flujos durante su transporte y la configuración de la zona de depósito de los mismos (Caldiño et al., 2002). Además, sus resultados son muy importantes para establecer las posibles áreas afectadas y son un elemento esencial para generar mapas de riesgo (Hsu et al., 2010).
No obstante, partiendo de la base de que la topografía es fundamental a la hora de calibrar un modelo (Stevens et al., 2002; Bateman et al., 2007; Dávila et al., 2007), es importante destacar el error implícito que puede existir en los resultados de este tipo de modelizaciones en función de la precisión y de la resolución de los modelos digitales de elevación (DEM, por sus siglas en inglés) disponibles. Por otro lado, también hay que considerar cuáles son los objetivos de la simulación a realizar y qué tipo de escenario se pretende diseñar puesto que, por ejemplo, modelizaciones de fenómenos usando topografías posteriores al evento estudiado pueden ser la causa de depósitos falsos o desviaciones de flujo (Bateman et al., 2007). Además, hay que tener en cuenta que algunos de los valores de los parámetros requeridos por estos modelos como el esfuerzo de cedencia, el coeficiente de rugosidad y la concentración volumétrica de sedimento tienen efectos significativos en los resultados simulados (Hsu et al., 2010) y muchas veces son de complicado cálculo o de gran variabilidad incluso dentro de un mismo evento, donde la concentración y la reología de los materiales pueden variar significativamente y la dominancia de esfuerzos viscosos y turbulentos puede alternarse y dar lugar a oleadas de flujo o pulsaciones (FLO-2D Software INC., 2017). Así pues, la elección de uno u otro valor puede modificar substancialmente los resultados.

\section{LA CUENCA TZUNUNÁ}

La cuenca de Tzununá se encuentra localizada en el sector NNW del lago Atitlán, ubicado en la región sur-occidental de la República de Guatemala formando parte de la Sierra Madre del Sur (Figura 1). El territorio pertenece al municipio de Santa Cruz la Laguna (Departamento de Sololá) y su población es de 2089 habitantes (LópezMaldonado, 2014). Esta ha sido seleccionada de entre las diferentes cuencas que drenan al lago Atitlán por sus características, por su representatividad en la zona y por los datos topográficos disponibles.

\section{Contexto geológico}

La geología de la cuenca está estrechamente ligada a los tres ciclos volcánicos y sismo-estructurales que han afectado al sistema caldérico de Atitlán, denominados Atitlán I, II y III respectivamente. Estos ciclos controlaron el crecimiento de estratovolcanes, la generación de diversos episodios eruptivos de carácter silíceo y la formación de tres grandes calderas (Newhall, 1987). El primero de ellos se originó hace 14 m.a. y el último hace 1 m.a. y fue el que dio lugar, como consecuencia de la gran erupción de "Los Chocoyos" (Núñez-Álvarez y Martínez-Cobo, 2011a), al colapso de la cámara magmática y a la formación de la última caldera, ocupada actualmente por el lago Atitlán. Los materiales cuaternarios que afloran en la cuenca (Figura 1) son depósitos aluviales (Qal1/Qal2/Qal4) y coluviales (Qcol2), depósitos piroclásticos (Qpa4) y depósitos de flujos de lavas, lodos y piroclastos (QTa). De forma aislada también aparecen niveles terciarios de metasedimentos asociados al relleno de caldera del ciclo Atitlán II (Tcf2) (Figura 1) (Núñez-Álvarez y Martínez-Cobo, 2011a, 2011b).

\section{Contexto geomorfológico}

La extensión de la cuenca de Tzununá es de $7.5 \mathrm{~km}^{2}$, su cota más baja se sitúa a $1563 \mathrm{~m}$, a nivel del lago Atitlán, y su punto más elevado a $2782 \mathrm{~m}$. La cota inferior oscila en función de las variaciones estacionales de la altura de la lámina de agua del lago. La longitud del río principal es de $3.8 \mathrm{~km}$, con un desnivel acumulado de $1219 \mathrm{~m}$. Su morfología, el pronunciado relieve y las pendientes elevadas son consecuencia directa de la formación de la caldera y de la intensa actividad fluviotorrencial a la que queda sujeta durante la época de lluvias. La superficie de la cuenca está subdividida en cuatro subcuencas (Figura 1). Solo una de 
ellas, la subcuenca 4, presenta circulación de aguas perennes a lo largo de su cauce principal. La red hidrológica es de tipo dendrítico en las zonas altas y de tipo trenzado en las zonas bajas de abanico.

Fenómenos hidrometeorológicos registrados en el área de estudio

Durante las dos últimas décadas, la zona del lago Atitlán se ha visto afectada por los ciclones tropicales Mitch (1998), Stan (2005) y Agatha (2010) y por diversos episodios de lluvias intensas de menor magnitud (Figura 2). Estos fenómenos llegaron a generar cantidades de precipitación acumulada en dos días consecutivos de $383 \mathrm{~mm}$ en el caso del huracán Stan en el 2005 y de $269 \mathrm{~mm}$ en el caso de la tormenta tropical Agatha en el 2010, y fueron los factores desencadenantes de numerosos movimientos de ladera y flujos torrenciales que afectaron a la región. El huracán Stan se formó el 1 de octubre de 2005 en la costa este de la península de Yucatán y avanzó en dirección oeste cruzándola y atravesando el golfo de México hasta deshacerse, el día 5 de octubre, en el estado de Oaxaca (México). Agatha se formó delante de la costa pacífica guatemalteca el 29 de mayo de 2010, avanzó hacia el continente en dirección noreste y acabó deshaciéndose sobre los territorios de la Sierra Madre del Sur el día 30 del mismo mes. En Tzununá, ambos ciclones provocaron el desbordamiento del río que transcurre a lo largo del abanico aluvial ocasionando graves daños en las viviendas ubicadas en las zonas afectadas.

\section{Procesos geomorfológicos asociados al paso de los ciclones tropicales Stan y Agatha}

Como consecuencia del paso del huracán Stan en el 2005 se contabilizaron, mediante el análisis de las fotografías aéreas, un total de 373 movimientos de ladera y flujos torrenciales sobre la superficie que cubre la cuenca de Tzununá. Teniendo en cuenta su extensión, la densidad de movimientos en la zona fue de 49 por $\mathrm{km}^{2}$. En la Figura 4 se puede comprobar cómo la gran mayoría de zonas de inicio de movimientos de ladera y flujos torrenciales se localiza en los sectores

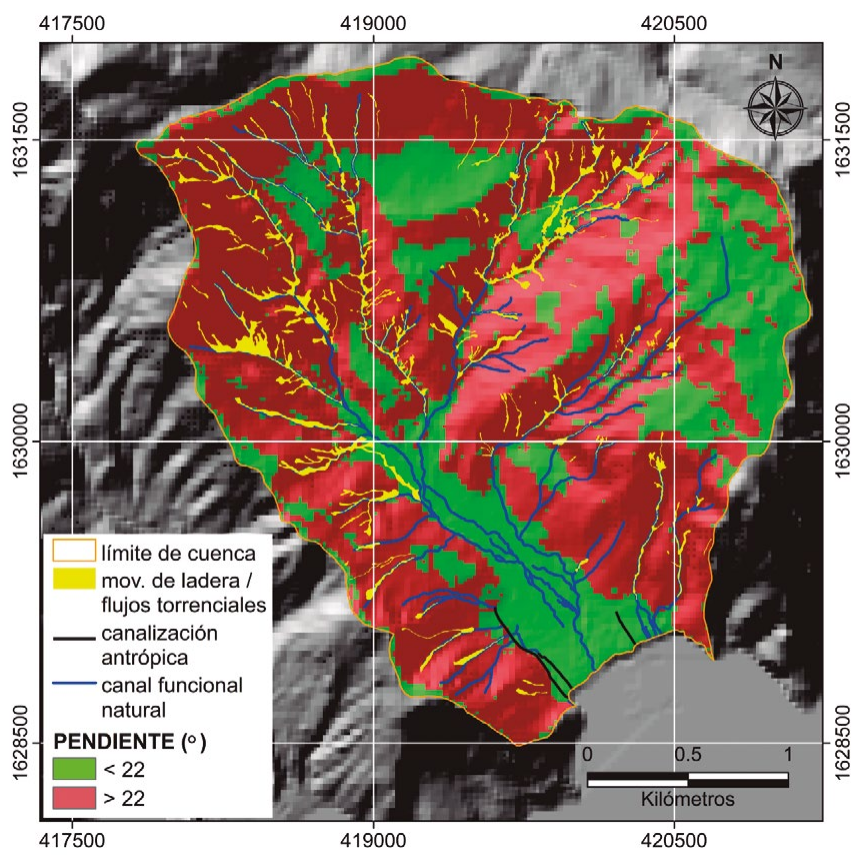

Figura 4. Mapa de pendientes y localización de la red hidrológica y de los movimientos de ladera y flujos torrenciales generados por el huracán Stan-2005. Sistema geográfico de referencia: GTM (Guatemala Transversa Mercator) / Datum: WGS 1984. con pendientes superiores a $22^{\circ}$, los cuales abarcan el $68 \%$ de la superficie total de la cuenca. En la zona de abanico aluvial el flujo principal desbordó generando un depósito de material de $0.03 \mathrm{~km}^{2}$. Durante el paso de la tormenta tropical Agatha (2010) se dieron un total de 17 movimientos de ladera y flujos torrenciales, 2.2 movimientos por $\mathrm{km}^{2}$. En este caso el flujo generado, de carácter más acuoso que el que generó Stan, socavó la base de uno de los muros de gaviones construidos posteriormente al año 2005 (Figuras 3a, 3b y 5). La estructura volcó hacia el lecho del río perdiendo eficacia y provocando el desbordamiento aguas abajo de este. El punto de desbordamiento coincidió con el punto por donde también desbordó el flujo generado por Stan en el 2005.

\section{METODOLOGÍA}

La metodología desarrollada ha estado condicionada por la escasez de datos y por la baja calidad de los mismos, ambos aspectos muy habituales en este tipo de contextos socioeconómicos. Aun así, se ha recopilado la mayor cantidad de información disponible y se ha tratado de optimizar para cumplir con los objetivos del trabajo y ofrecer los resultados que se presentan más adelante. De esta manera, la elección de los valores de precipitación acumulada durante la tormenta tropical Agatha en el 2010 se ha realizado teniendo en cuenta la fiabilidad y continuidad de los mismos ya que los registros de los valores de precipitación de otros fenómenos meteorológicos, como por ejemplo los del huracán Stan, no son continuos o presentan algunas imprecisiones por lo que su utilización no ha sido viable. A los valores de caudal resultantes, calculados a partir de dichas precipitaciones, se les han añadido diferentes porcentajes de carga sólida aumentando así su volumen y generando flujos de mayor magnitud. Teniendo en cuenta este tipo de escenarios, similares a los acontecidos durante Stan, y la disponibilidad de material gráfico posterior a ambos fenómenos, se considera interesante comparar los resultados obtenidos tanto con los efectos producidos por Stan (2005) como por Agatha (2010).

\section{Cartografía geomorfológica de indicadores antecedentes}

La cartografía geomorfológica de indicadores antecedentes se ha realizado mediante tres pasos:

1. Recopilación de información existente y análisis de fotografías aéreas.

2. Observaciones y mediciones de campo.

3. Recopilación de datos y cartografía de indicadores geomorfológicos en un SIG.

Se llevó a cabo una recopilación de trabajos previos realizados en la zona de estudio por Núñez-Álvarez y Martínez-Cobo (2011a, 2011b), Paricio-Salas (2011), Serra-Creixell (2011) y Hernández et al. (2012) y una fotointerpretación detallada para identificar morfologías asociadas a los fenómenos hidrogeomorfológicos ocurridos. Para ello, se han utilizado las fotografías aéreas de los vuelos realizados en los años 2006 y 2011, ambos posteriores a los fenómenos hidrometeorológicos tomados como referencia. Durante el trabajo de campo se ha realizado un reconocimiento de las zonas de inicio de movimientos de ladera y flujos torrenciales, de los puntos de desbordamiento y de los depósitos generados en las zonas de llegada. También se han geolocalizado y delimitado todos los indicadores antecedentes y elementos geomorfológicos y morfodinámicos observados: canales funcionales principales y secundarios, canalizaciones antrópicas, superficie de abanico aluvial, frentes de paleodesembocadura, zonas de inicio, trayecto y llegada de movimientos de ladera y flujos torrenciales, divisorias de agua principales (parteaguas) y secundarias (crestas de depósito), zonas erosivas y deposicionales y niveles históricos del lago. Además, se ha realizado una cartografía detallada de la distribución 


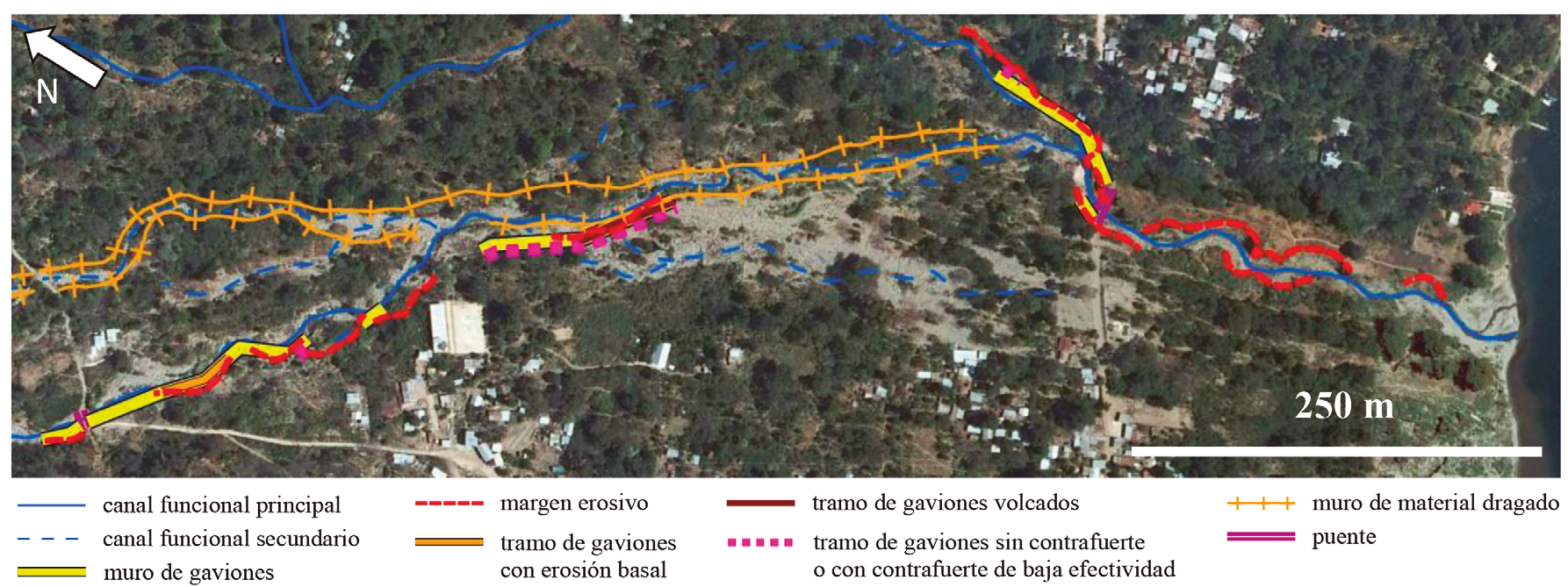

Figura 5. Mapa de tipología de muros y márgenes. Ampliación del tramo bajo del canal principal (ver Figura 1d).

y tipología de los muros levantados a lo largo de los márgenes del río principal en su tramo final (Figura 5). Paralelamente se ha recopilado información testimonial de los habitantes de la zona que ha sido de especial importancia para describir la dinámica y para cuantificar la magnitud y tipología de los procesos analizados. Los datos recogidos y la información generada se han integrado y representado en un mapa geomorfológico de indicadores antecedentes (MGIA) (ver apartado de "resultados"). Finalmente se han hecho observaciones y se han recogido datos durante periodos de lluvias intensas acontecidos en la zona. Esto ha ayudado a entender de forma más clara la dinámica de los fenómenos y a obtener datos reales in situ utilizados posteriormente para validar los resultados de las modelizaciones.

\section{Modelización con FLO-2D}

El software FLO-2D, desarrollado por O'Brien et al., (1993), es un modelo bidimensional de diferencias finitas aprobado por la FEMA (Federal Emergency Management Agency) como modelo hidráulico (FLO-2D Software INC., 2017) y concebido para modelizar avenidas y flujos de diferentes características sobre una superficie de terreno previamente delimitada. El modelo destaca, respecto a otros, tanto por la posibilidad de simular flujos con porcentajes variables de carga sólida de diferentes propiedades reológicas como por su carácter bidimensional. Además de ofrecer información del recorrido del flujo teniendo en cuenta ocho direcciones potenciales de avance, da una predicción de altura de flujo acumulada y de velocidad para cada una de las celdas que conforman la malla sobre la que se desarrolla la modelización. Cabe destacar que la predicción de altura de flujo no es siempre equiparable a zonas de acumulación de detritos, sino que representa la máxima profundidad de flujo independientemente de si se generan o no depósitos de material.

Los parámetros principales que utiliza para su desarrollo son: a) la topografía, b) los valores de precipitación/caudal, c) el porcentaje de concentración de carga sólida, d) los valores del coeficiente de Manning (n), e) los parámetros reológicos y la granulometría de los sedimentos y detritos transportados y f) el número de Froude. Para la integración de toda la información necesaria se utiliza el pre-procesador GDS (Grid Developer System), a partir del cual se genera una malla que permite asignar a cada una de las celdas que la componen los diferentes valores y datos disponibles. En el presente estudio se ha utilizado la versión 2011 de FLO-2D.

La información topográfica para la modelización se generó a partir de un DEM con una resolución de $15 \times 15 \mathrm{~m}$ junto con una topografía de detalle del cauce del canal funcional principal de $5 \times 5 \mathrm{~m}$ de resolución, obtenida a partir de un levantamiento topográfico con estación total. Estas dos fuentes de datos espaciales se han integrado en una sola a partir del software MICROSTATION (Bentley Systems) para obtener una topografía de detalle del abanico aluvial y, en consecuencia, una mayor precisión en los resultados finales. La correcta integración de ambas fuentes de datos se ha contrastado y corregido comparando las dimensiones y el calado de secciones reales levantadas en campo con diversas secciones del DEM integrado.

Los valores de precipitación utilizados se han obtenido de los datos de intensidad horaria registrados por el INSIVUMEH (2013) (Instituto Nacional de Sismología, Vulcanología, Meteorología e Hidrología de Guatemala) durante la tormenta tropical Agatha en el 2010 en la estación meteorológica de Santiago de Atitlán, situada a $11.5 \mathrm{~km}$ de la zona de estudio (1b). El cálculo de la precipitación efectiva y de los valores de caudal resultante se ha realizado mediante el software HEC-HMS (US Army Corps of Engineers). Para el cálculo de pérdidas y de precipitación efectiva se ha utilizado el método del número de curva $(\mathrm{CN})$ desarrollado por el NRCS (Natural Resources ConservationService) (Hernández et al., 2012). Para la transformación del exceso de precipitación (precipitación efectiva) en escorrentía superficial se ha empleado el método del hidrograma unitario desarrollado también por el NRCS. Este requiere solo de un parámetro y es ideal cuando se cuenta con escasa información (Hernández et al., 2012). Teniendo en cuenta el tipo de escenarios climatológicos modelizados, las pérdidas por evaporación se han despreciado y el flujo base se ha considerado no significativo respecto a los caudales de avenida.

Los valores de caudal resultantes son, consecutivamente, los derivados de las cuatro horas de máxima precipitación registrada durante la tormenta tropical Agatha: 12, 22.4, 36.2 y $10.5 \mathrm{~m}^{3} / \mathrm{s}$ (Figura 6). Estos valores han sido los utilizados como valores base en los tres escenarios simulados y corresponden al periodo de máxima descarga. La ausencia de datos fiables de referencia respecto a las concentraciones volumétricas de carga sólida en los flujos acontecidos en el pasado ha llevado a la definición de tres escenarios de modelización hipotéticos con el objetivo de abarcar el rango de fenómenos más probables en función de las observaciones de campo y de las características de los fenómenos antecedentes. En el primero se ha modelizado un flujo acuoso sin carga sólida añadida. En el segundo se ha modelizado un flujo con un 15\% en volumen de carga sólida añadido a los valores de 


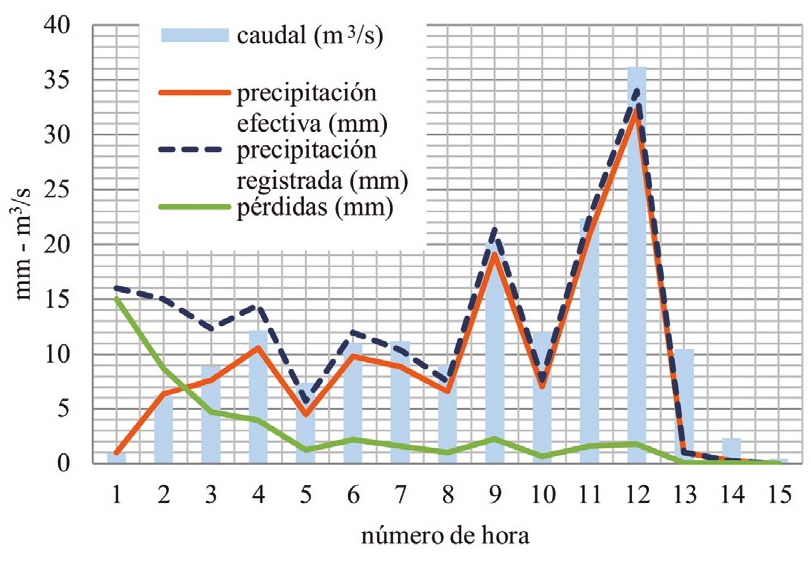

Figura 6. Representación gráfica de los valores de precipitación registrada, pérdidas, precipitación efectiva y caudal resultante de las precipitaciones que se dieron durante Agatha-2010. El eje-Y representa los valores tanto de precipitación y de pérdidas (en $\mathrm{mm}$ ) como de caudal (en $\mathrm{m}^{3} / \mathrm{s}$ ). El eje-X representa el número de horas de precipitación contabilizadas para los cálculos. La modelización se ha desarrollado entre las horas 10 y 13.

caudal base. Este porcentaje es el representativo de la carga de fondo y de la carga en suspensión (opción bulking concentration). En el tercero se ha modelizado un flujo con un porcentaje en volumen de carga sólida añadido al caudal base para cada una de las cuatro horas de la modelización (opción mud and debris). Este ha sido del 10, 20, 45 y $10 \%$ para cada hora respectivamente. Según la clasificación propuesta anteriormente, los dos primeros escenarios entrarían dentro del campo de los flujos acuosos (flujos con un porcentaje de carga sólida $<20 \%$ ) y el tercer escenario correspondería a un flujo hiperconcentrado (flujo con un porcentaje de carga sólida comprendido entre el 20 y el $60 \%)$.

Los valores del coeficiente de Manning (n) asociados a cada celda de cálculo se han obtenido a partir de la propuesta de FernándezLavado (2010) realizada para contextos geológicos y geomorfológicos similares a los de la zona de estudio. La relación de estos valores en función de los distintos usos del suelo se ha llevado a cabo mediante su correlación con el mapa de usos del suelo elaborado por el MAGA (Ministerio de Agricultura, Ganadería y Alimentación de Guatemala) (2010) (Tabla 1).

Los parámetros reológicos de los materiales implicados se han extraído de los ensayos realizados por Oliva-Hernández (2007) (Tabla 2) en otra de las cuencas que rodean el lago Atitlán ya que los materiales utilizados en dichos ensayos son litológicamente similares, y de características reológicas análogas a los presentes en la cuenca de Tzununá.

Tabla 1. Valores del coeficiente de Manning (n) escogidos en función de los usos del suelo de la zona de estudio.

\begin{tabular}{lc}
\hline Uso del suelo & $\begin{array}{c}\text { Valor del Coef. de Manning } \\
(\mathbf{n})\end{array}$ \\
\hline Tejido urbano & 0.015 \\
Espacios abiertos sin o con poca vegetación & 0.03 \\
Cultivos anuales & 0.035 \\
Cultivos permanentes & 0.04 \\
Vegetación arbustiva baja & 0.05 \\
Ríos & 0.055 \\
\hline
\end{tabular}

De acuerdo con FLO-2D Software INC. (2017), y partiendo de que los flujos que se dan en la zona de estudio son de régimen subcrítico y se transportan a bajas velocidades sobre superficies de abanico aluvial con pendientes moderadas, se ha establecido un valor del número de Froude de 0.85 .

\section{RESULTADOS}

Los resultados del trabajo realizado se presentan en cuatro bloques: 1. El Mapa Geomorfológico de Indicadores Antecedentes (MGIA) (Figura 7).

2. El Mapa de Susceptibilidad a Inundación elaborado a partir del método geomorfológico integrado (MSI) (Figura 8).

3. Los resultados obtenidos en las modelizaciones de los tres escenarios diseñados (Figuras 9, 10 y 11).

4. La integración de los resultados de ambas metodologías en un Mapa Integrado de Susceptibilidad a Inundación (MISI) (Figura 12).

Mapa Geomorfológico de Indicadores Antecedentes - MGIA

Los pasos seguidos para la elaboración de este mapa y los diferentes elementos o indicadores cartografiados se han descrito ampliamente en el subapartado de la metodología "Cartografía geomorfológica de indicadores antecedentes".

\section{Mapa de susceptibilidad a inundación - MSI (Método Geomorfológico integrado)}

La elaboración del MSI (Figura 8) se ha basado en el análisis multicriterio y en la integración de la información aportada a través del trabajo de gabinete, del trabajo de campo y de su combinación, representada en el Mapa Geomorfológico de Indicadores Antecedentes (MGIA). Este es de carácter cualitativo y para su representación se han considerado tres grados de susceptibilidad a inundación. Estos niveles definen la posibilidad de las áreas estudiadas (alta, baja y media) a ser afectadas de nuevo por inundaciones si se repitieran flujos de características similares a los que se dieron durante los eventos de Stan (2005) y Agatha (2010). Los diferentes sectores zonificados cubren la extensión correspondiente a la superficie de abanico representada en el mapa de indicadores antecedentes.

Los sectores con susceptibilidad a inundación alta corresponden a las zonas de circulación de los canales funcionales principales y secundarios y a las zonas de abanico aluvial que se han visto afectadas por fenómenos de inundación, ya sea por flujos acuosos o por flujos hiperconcentrados. Los puntos de desbordamiento de los flujos suelen concentrarse en los tramos donde el cauce del río cambia de dirección. En estos tramos, tanto la actividad erosiva como la deposicional son muy intensas y provocan importantes y repentinos cambios en la

Tabla 2. Parámetros reológicos utilizados para la modelización de flujos hiperconcentrados (Fuente: Oliva-Hernández, 2007).

\begin{tabular}{|c|c|}
\hline $\begin{array}{l}\text { Viscosidad dinámica } v s . \\
\text { concentración volumétrica }\end{array}$ & $\begin{array}{l}\text { Esfuerzo cortante de cedencia } v s \text {. } \\
\text { concentración volumétrica }\end{array}$ \\
\hline$y=0.030 e^{26.39 x}$ & $y=0.005 e^{32.45 x}$ \\
\hline$\beta=26.39$ & $\beta=32.45$ \\
\hline Gravedad específica & Resistencia al flujo laminar (k) \\
\hline 2.6 & 400 \\
\hline
\end{tabular}




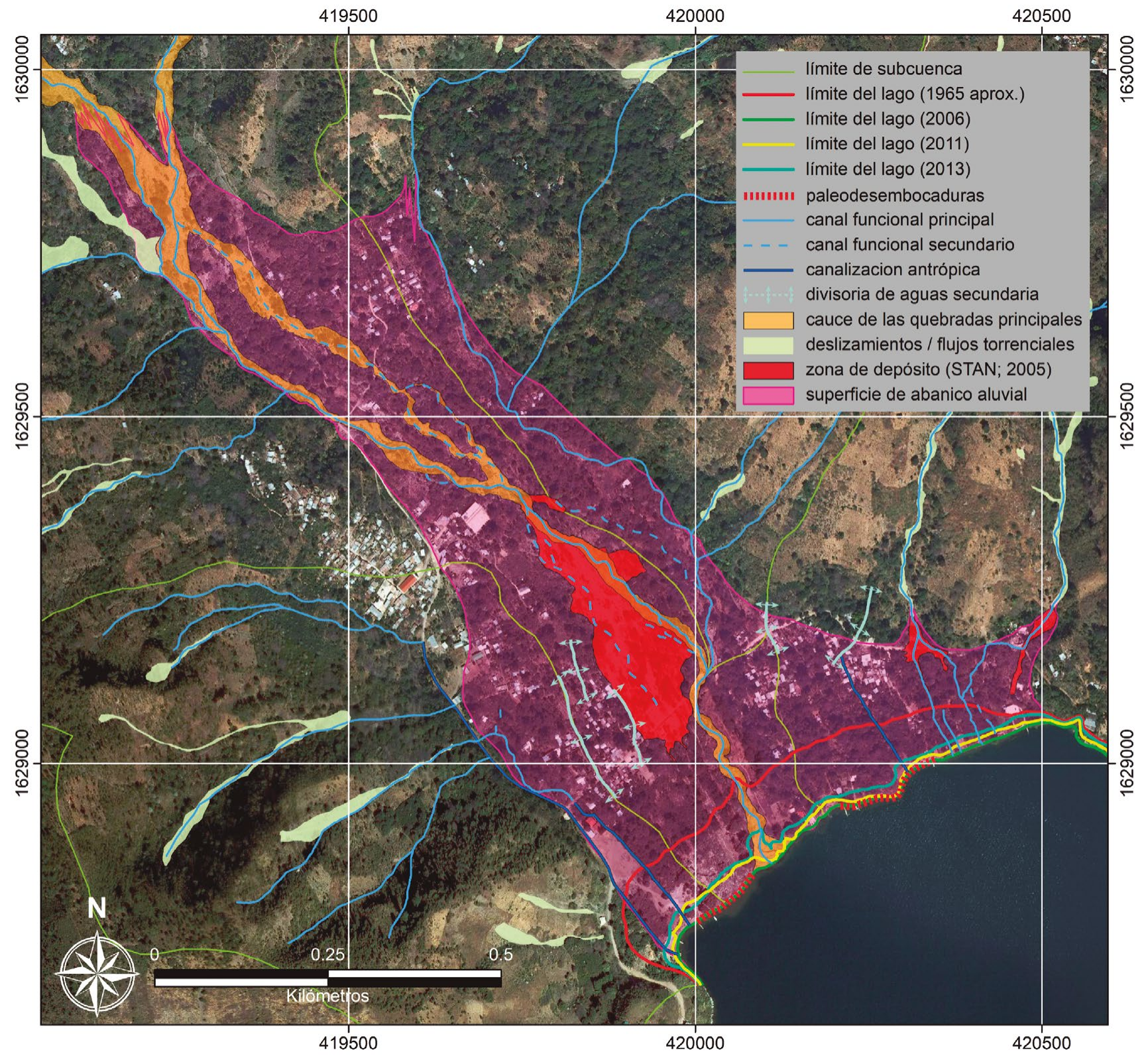

Figura 7. Mapa Geomorfológico de Indicadores Antecedentes (MGIA). Sistema geográfico de referencia: GTM (Guatemala Transversa Mercator) / Datum: WGS 1984.

morfología y en la capacidad del lecho fluvial. Por un lado, la erosión lateral-basal del cauce desestabiliza los márgenes y, por el otro, la acumulación de materiales disminuye su capacidad portante facilitando, en ambos casos, el desbordamiento. La extensión con susceptibilidad alta abarca el $74.5 \%$ del total de la superficie zonificada, cubriendo una extensión total de $0.35 \mathrm{~km}^{2}$. Para realizar la delimitación de los sectores con susceptibilidad media y baja se ha tenido en cuenta su grado de proximidad a canales funcionales principales y secundarios, la presencia de canalizaciones antrópicas, la localización de pequeñas crestas de depósito a lo largo del terreno y la existencia de zonas más elevadas topográficamente. Este tipo de morfologías se forman como consecuencia de la dinámica histórica de erosión/sedimentación del abanico y condicionan el comportamiento distal de futuros flujos. Las zonas de susceptibilidad media y baja abarcan el 14.8 y el $10.7 \%$ de la superficie zonificada y cubren una extensión total de 0.07 y $0.05 \mathrm{~km}^{2}$ respectivamente.

\section{Modelización con FLO-2D}

\section{Escenario 1 (flujo acuoso sin carga sólida)}

En el escenario 1 se ha modelizado un caudal acuoso sin carga sólida correspondiente al caudal base obtenido con el software HECHMS. El volumen total de flujo generado ha sido de $273060 \mathrm{~m}^{3}$, la profundidad de flujo máxima registrada ha sido de $1.9 \mathrm{~m}$ y la velocidad máxima de $4.7 \mathrm{~m} / \mathrm{s}$. Los resultados de la modelización muestran coincidencias con uno de los puntos de desbordamiento (Punto 1 en Figura 9) que se produjo tanto durante el paso de Stan como de Agatha. Aguas abajo, en la zona donde el canal principal muestra un cambio brusco de dirección de SE hacia SW, el modelo marca de nuevo otro punto de desbordamiento que no se dio ni durante el paso de Stan ni de Agatha (Punto 2 en Figura 9). Esto podría deberse a un valor erróneo de altura asignado a las celdas del GDS o a que durante el paso de ambos ciclones por la región la morfología y profundidad 


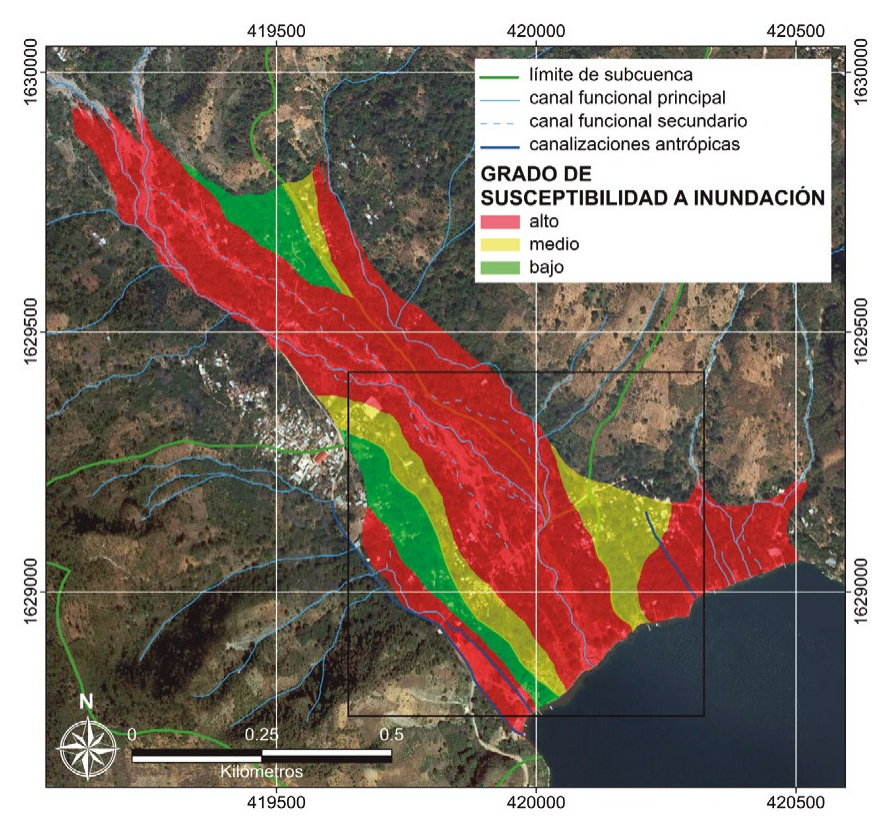

Figura 8. Mapa de Susceptibilidad a Inundación (MSI). El recuadro negro indica la zona representada en las Figuras 9, 10 y 11. Sistema geográfico de referencia: GTM (Guatemala Transversa Mercator) / Datum: WGS 1984.

del canal en ese punto era diferente a la actual. No obstante, teniendo en cuenta el marcado cambio de dirección del canal, dicho sector se considera crítico puesto que es altamente vulnerable debido a la importante acción erosiva a la que se encuentra expuesto por impacto frontal del flujo. Por otro lado, si extendemos virtualmente el recorrido del canal en dirección NW-SE este acabaría desembocando en una de las paleodesembocaduras representadas en el mapa de indicadores antecedentes (Punto 4 en Figura 9). Esto sugiere que dicha extensión ya

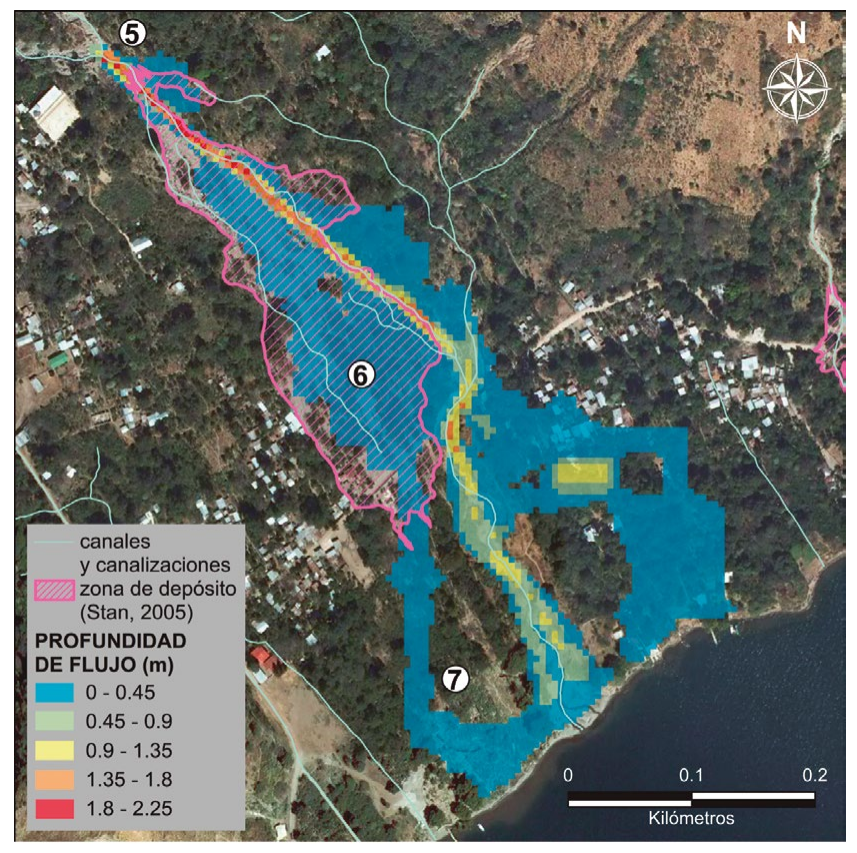

Figura 10. Profundidades de flujo máximas acumuladas en la modelización del escenario 2.

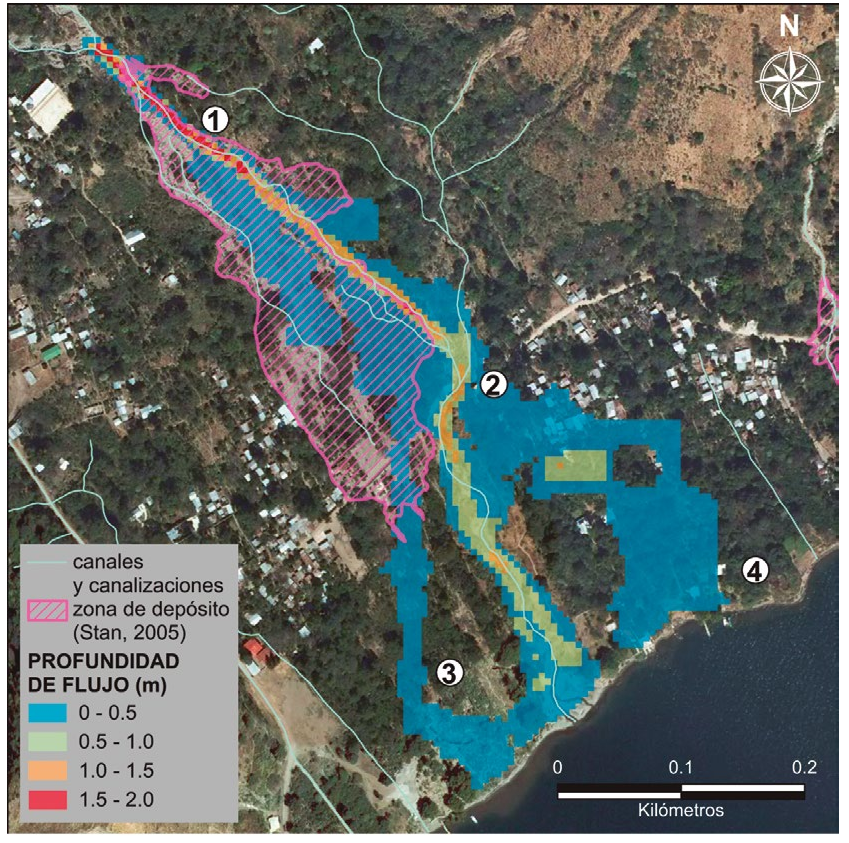

Figura 9. Profundidades de flujo máximas acumuladas en la modelización del escenario 1.

habría sido ocupada en el pasado por canales lo que, a su vez, indicaría un mayor grado de susceptibilidad a afectación por futuros procesos. Finalmente, los modelos señalan un avance del flujo en dirección N-S (Punto 3 en Figura 9). Teniendo en cuenta que la distribución de las celdas del DEM y el recorrido del flujo muestran la misma orientación, dicho avance podría explicarse por la existencia de algún dato erróneo de elevación que habría dado lugar a una hondonada en el terreno. No obstante, el final de la zona de avance del flujo acaba coincidiendo de

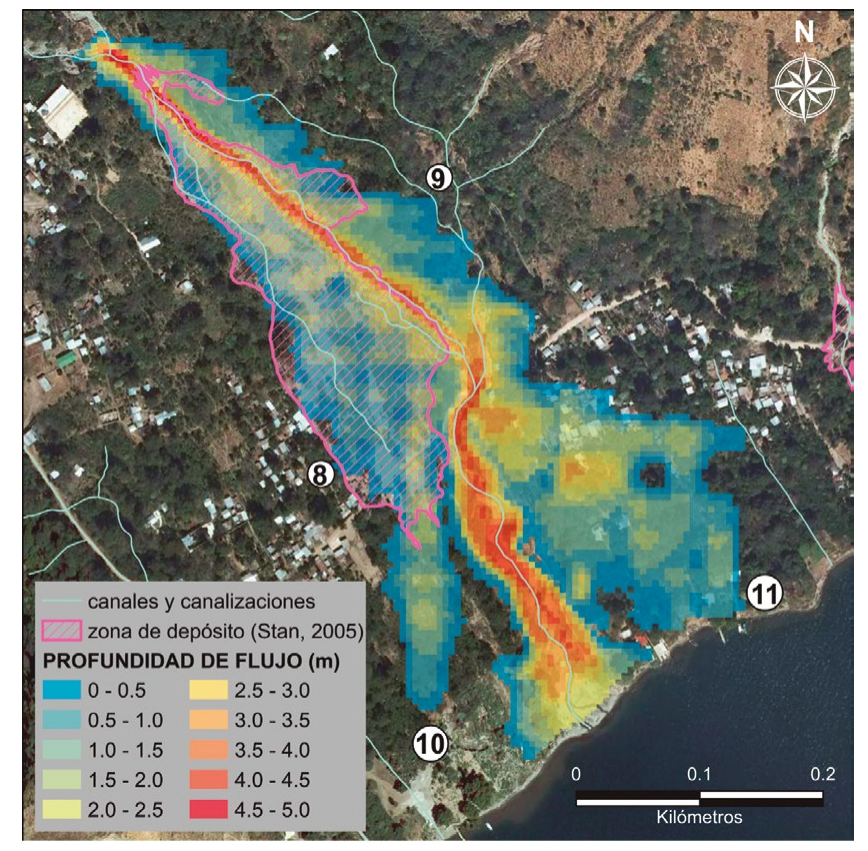

Figura 11. Profundidades de flujo máximas acumuladas en la modelización del escenario 3 . 
nuevo con otra de las paleodesembocaduras identificadas en el análisis geomorfológico por lo que, aunque el recorrido del flujo modelado puede no corresponderse exactamente con la trayectoria real, los indicadores recogidos señalan que la zona también tiene un grado de susceptibilidad elevado a ser afectada por futuros flujos torrenciales.

\section{Escenario 2 (flujo con carga de fondo y en suspensión)}

En el escenario 2 se ha modelizado un flujo con un incremento de volumen del $15 \%$ respecto al caudal base utilizando la opción de FLO-2D bulking concentration. En nuestro caso, un incremento de la concentración volumétrica del $15 \%$ genera un factor de 1.18 que, multiplicado por los caudales de referencia obtenidos, da un resultado de caudal final para cada una de las horas de modelización de 14.2, $26.4,42.7$ y $12.4 \mathrm{~m}^{3} / \mathrm{s}$ respectivamente. Este incremento representa un flujo acuoso con carga de fondo y con carga en suspensión. Este tipo de flujos es frecuente durante la época de lluvias y se da como consecuencia de pequeñas crecidas de los ríos derivadas de lluvias muy intensas. El volumen total de flujo generado ha sido de $321252 \mathrm{~m}^{3}$, la profundidad de flujo máxima registrada ha sido de $2.1 \mathrm{~m}$ y la velocidad máxima de $4.9 \mathrm{~m} / \mathrm{s}$.

En la modelización se observa un nuevo punto de desbordamiento localizado en el margen izquierdo del río (Punto 5 en Figura 10), aguas arriba del punto 1 . Este coincide con un sector donde también se produjo un desbordamiento durante el paso del huracán Stan y donde, además, se formó un pequeño depósito. También se observa cómo la zona afectada por el desbordamiento del punto 1 se amplía debido al incremento del caudal llegando casi a cubrir completamente la extensión de terreno que abarca el depósito de material que generó el flujo acontecido durante el paso del huracán Stan (Punto 6 en Figura 10). Finalmente, de la misma manera que en el escenario 1 , también se produce el avance del flujo en dirección N-S hacia la paleodesembocadura situada más al sur del abanico (Punto 7 en Figura 10).

\section{Escenario 3 (flujo hiperconcentrado)}

En el escenario 3 se ha modelizado un flujo con un pico máximo de concentración volumétrica de sedimento del $45 \%$. El volumen total de flujo generado ha sido de $390494 \mathrm{~m}^{3}$, la profundidad de flujo máxima registrada ha sido de $4.9 \mathrm{~m}$, la velocidad máxima de $4.3 \mathrm{~m} / \mathrm{s}$ y la velocidad máxima en el momento de mayor contenido de carga sólida, y por lo tanto de mayor viscosidad, de $1.4 \mathrm{~m} / \mathrm{s}$.

Los resultados de la modelización muestran una clara coincidencia entre la extensión del depósito generado por los flujos derivados del paso del huracán Stan y la extensión que abarca el flujo hiperconcentrado simulado a lo largo del margen derecho del canal principal (Punto 8 en Figura 11). En el margen izquierdo también aumenta la extensión abarcada por el flujo respecto a los escenarios 1 y 2 . En este caso, y aunque durante el trabajo de cartografía no se identificaron restos de depósitos, el límite situado más al NE coincide con un canal secundario que sí se pudo cartografiar y que acaba conectando con otro canal tributario del canal principal (Punto 9 en Figura 11). Tal y como se observa en el punto 10, es importante destacar como, a diferencia de los escenarios 1 y 2, los resultados de esta modelización no muestran que el flujo llegue a desembocar en el lago. Esto se debe probablemente a su alta viscosidad consecuencia tanto de la elevada concentración de carga sólida como de sus características reológicas. Según Julien et al. (1991) concentraciones volumétricas de entre 15 y $45 \%$ de sólidos pueden incrementar la viscosidad dinámica de un flujo entre 1.5 y 4 órdenes de magnitud respecto a un flujo acuoso. En el margen izquierdo de la canalización el flujo modelizado desemboca en el lago aumentando la extensión afectada respecto a los escenarios anteriores en ese sector (Punto 11 en Figura 11).

\section{Mapa Integrado de Susceptibilidad a Inundación - MISI}

La elaboración del MISI (Figura 12) se ha realizado en función de los resultados de la MSI y de los mapas de extensión y profundidad de

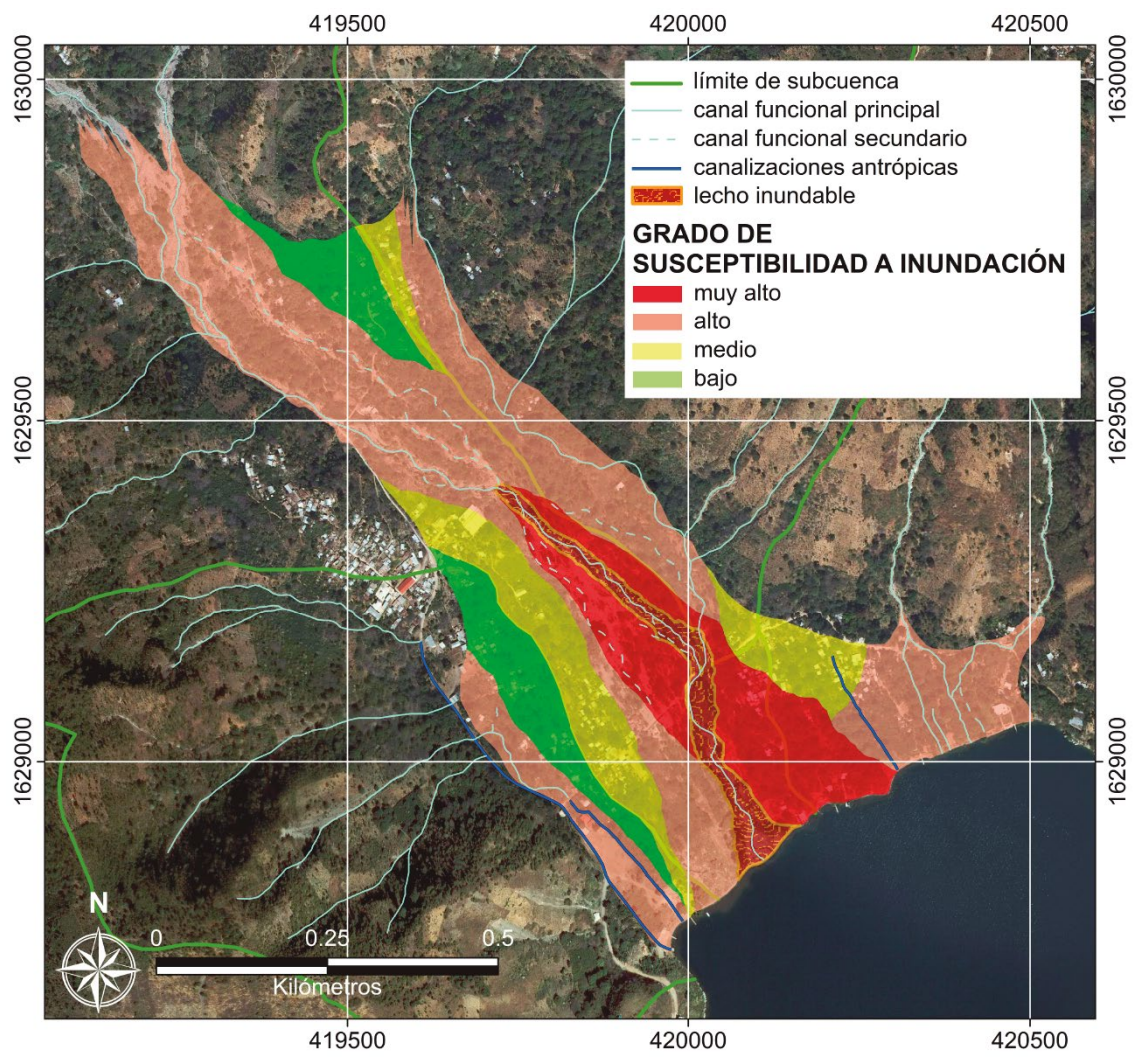

Figura 12. Mapa Integrado de Susceptibilidad a Inundación (MISI) desarrollado a partir del método geomorfológico y de la modelización con FLO-2D. Sistema geográfico de referencia: GTM (Guatemala Transversa Mercator) / Datum: WGS 1984. 
flujo obtenidos como resultado de las modelizaciones con el software FLO-2D. La redistribución espacial de los polígonos de susceptibilidad llevada a cabo respecto al MSI se localiza solamente en la mitad topográficamente más baja del abanico aluvial debido a las limitaciones espaciales propias de la extensión de la modelización.

La información recogida y generada ha permitido establecer inicialmente un límite de lecho inundable que abarca un $4.9 \%$ de la superficie zonificada y tiene una extensión de $0.02 \mathrm{~km}^{2}$. Sus márgenes se han definido a partir de la topografía de detalle del cauce y de las zonas donde la profundidad de flujo acumulada en las modelizaciones ha superado los $2 \mathrm{~m}$. Además se ha establecido un grado más de susceptibilidad denominado "muy alto" delimitado esencialmente a partir de los límites de la extensión del flujo resultante de la modelización del escenario 3. Su superficie abarca un $13.5 \%$ del total y se extiende a lo largo de $0.06 \mathrm{~km}^{2}$. Esta nueva gradación ha permitido dividir en "alto" y "muy alto" el grado de susceptibilidad definido en la MSI como "alto". Finalmente, y teniendo en cuenta el análisis de los resultados llevado a cabo en los sectores de los puntos 3 y 4 de la Figura 9 y del punto 7 de la Figura 10, se han realizado ajustes en la extensión espacial de los polígonos de susceptibilidad media y baja disminuyendo su extensión y ampliando las zonas definidas con un grado de susceptibilidad mayor. Estos ajustes se concentran principalmente en el sector más cercano al lago a ambos lados del canal principal. El porcentaje de superficie ocupado por los grados alto, medio y bajo es, en el mismo orden, del $56.2,15.1$ y $10.3 \%$ ocupando una extensión de $0.27,0.07$ y $0.05 \mathrm{~km}^{2}$ respectivamente.

\section{DISCUSIÓN Y CONCLUSIONES}

Considerando el grado de presión socio-urbanística a la que están sometidas determinadas zonas del planeta, la elaboración de mapas de susceptibilidad a inundación por flujos torrenciales es una herramienta necesaria y esencial para facilitar un desarrollo ordenado y acorde con las características y las dinámicas geomorfológicas y climáticas de cada región.

Los fenómenos analizados en el presente trabajo están controlados por una gran cantidad de factores. Muchos de ellos son extremadamente difíciles de cuantificar y de definir y, a su vez, presentan una amplia variabilidad incluso dentro de un mismo evento. Este hecho hace que debamos asumir de antemano las propias limitaciones derivadas de la complejidad de los procesos y la dificultad e incertidumbre asociada en lo referente al análisis de su comportamiento y magnitud. La integración de las dos metodologías desarrolladas para tal efecto posibilita una mejora notable en la precisión de los resultados de predicción espacial de alcance. En ese sentido es especialmente relevante el análisis geomorfológico realizado, tanto por su nivel de detalle como por los datos clave que ha aportado. A diferencia de otros estudios centrados únicamente en la modelización hidráulica de este tipo de fenómenos, el desarrollo paralelo de un trabajo de campo preciso y exhaustivo permite compensar las limitaciones implícitas de los resultados de los modelos numéricos. Esto se realiza a partir de la recopilación de toda una serie de indicadores dejados por los fenómenos antecedentes acontecidos durante Stan (2005) y Agatha (2010) y del estudio pormenorizado in situ de las características geomorfológicas de la zona. Ambos son factores determinantes que posibilitan el examen de los resultados de las modelizaciones desde un punto de vista crítico y selectivo. Además, nos proporcionan herramientas para dar mayor o menor peso a los resultados y, así, validar o descartar los valores obtenidos.

Tal y como hemos visto al comparar los resultados obtenidos, la mayor parte de las áreas afectadas por flujos en las modelizaciones se correlacionan con las áreas afectadas por flujos torrenciales antecedentes. Este buen nivel de correspondencia ha favorecido la utilización de dichos resultados con un grado de confianza razonable y su integración en el Mapa Integrado de Susceptibilidad a Inundación (MISI). A su vez, también ha posibilitado la discretización de los valores de susceptibilidad representados en el Mapa de Susceptibilidad a Inundación (MSI) y ha dado mayor peso y fiabilidad en el momento de establecer determinadas limitaciones espaciales. Los resultados de profundidad de flujo acumulado son representativos de fenómenos potenciales y muestran un buen grado de coherencia con los datos topográficos de la zona de abanico aluvial y el calado y la capacidad hidráulica de las canalizaciones. Los valores muestran máximos de profundidad de flujo de entre 1.9 y $2.1 \mathrm{~m}$ para los escenarios 1 y 2 respectivamente y de $4.9 \mathrm{~m}$ para el escenario 3 . Los datos de velocidad máxima de entre 4.3 y $4.9 \mathrm{~m} / \mathrm{s}$ también son razonables y encajan perfectamente con algunos de los valores registrados in situ en la región durante crecidas ordinarias y extraordinarias. En ese sentido, la disminución progresiva de la velocidad del flujo del escenario 3, proporcional al aumento de carga sólida, es coherente y también encaja perfectamente con los valores esperables.

No obstante, algunos de los resultados de distribución espacial de flujo generados a partir de las modelizaciones no se ajustan ni corresponden con las extensiones afectadas por los flujos antecedentes. Esto puede deberse a errores relacionados con la calidad y la representatividad de los datos base disponibles (DEM, precipitación y caudal), a la estimación del volumen de los flujos simulados o a las propias limitaciones de los modelos a la hora de representar la complejidad de este tipo de fenómenos a partir de los valores de entrada (coeficiente de Manning (n), valores reológicos y porcentaje de carga sólida). En estos casos, el contraste entre los datos de ambas metodologías y su complementación son determinantes para el filtrado de los datos y la mejora en la precisión y la calidad de los mapas finales.

La utilidad a corto plazo de los mapas integrados de susceptibilidad a inundación resultantes debe centrarse en el diseño de sistemas de alerta temprana de mayor eficacia puesto que, en la mayoría de casos, la construcción de obras de mitigación adecuadas o la reubicación de parte de los núcleos urbanos más expuestos no son viables en términos socioeconómicos. En ese sentido, los actuales avances científico-técnicos en lo referente a la previsión meteorológica favorecen claramente este tipo de actuaciones. Teniendo esto en cuenta, y con el objetivo principal de evitar la pérdida de vidas humanas, las vías de trabajo e investigación deben focalizarse en la prevención, previsión y creación de protocolos de evacuación en las poblaciones o comunidades ubicadas en zonas con un alto grado de susceptibilidad a ser afectadas por fenómenos destructivos de tipo flujo torrencial.

\section{AGRADECIMIENTOS}

Esta investigación fue realizada con la financiación y el apoyo logístico de la organización Vivamos Mejor (Guatemala) y con el apoyo técnico de la Universitat de Barcelona (UB) y de la Universitat Autónoma de Barcelona (UAB). Queremos agradecer de manera especial la gran ayuda recibida por los y las habitantes de Tzununá sin los y las cuales no hubiera sido posible realizar los trabajos de campo que se presentan en el estudio. De la misma manera, su testimonio sobre los fenómenos acontecidos en el pasado ha sido esencial para poder caracterizar los procesos estudiados. Finalmente, agradecer a Juan Pablo Oliva Hernández, actual Director Ejecutivo del INSIVUMEH, el apoyo recibido en lo referente al trabajo con FLO-2D, a Elsa Benítez, Susan Kelly y Osman Konuray las aportaciones y mejoras propuestas sobre el texto y al equipo de revisores y revisoras el trabajo de revisión realizado. 


\section{REFERENCIAS}

Bateman, A., Medina, V., Hürlimann, M., Velasco, D., 2007, Modelo bidimensional para simulación de flujos detríticos: FLAT Model. Aplicación a una cuenca del Pirineo Catalán: Ingeniería hidráulica en México, XXII(4), 5-20.

Beven II, J.L., 2010, Archive of Hurricane Reports: Estados Unidos. National Oceanic and Atmospheric Administration (NOAA), National Hurricane Center (NHC), base de datos, <http://www.nhc.noaa.gov/>, acceso libre, consulta: 12 de mayo de 2017.

Beverage, J.P., Culberstone, J.K., 1964, Hyper concentrations of suspended sediment: American Society of Civil Engineers proceedings, Journal of the hydraulics division, 90, 117-128.

Brea, J.D., Spalletti, L., Hopwood, H.J., Spalletti, P.,2005, Conceptos y aplicaciones sobre flujos densos en la hidráulica de ríos: Ingeniería del agua, 12(3), 1-12.

Caballero, L., Capra, L., 2014, The use of FLO2D numerical code in lahar hazard evaluation at Popocatépetl volcano: a 2001 lahar scenario: Natural Hazards Earth System Sciences, 14, 3345-3355.

Caldiño, I.A., Bonola, I., Salgado, G., 2002, Determinación en el laboratorio de los parámetros reológicos de mezclas agua-sedimentos para el cálculo de flujos de lodos y debris: Ingeniería hidráulica en México, XVII(3), 27-35.

Capra, L., Borselli, L., Varley, N., Gavilanes-Ruiz, J.C., Norini, G., Sarocchi, D., Caballero, L., Cortes, A., 2010, Rainfall-triggered lahars at Volcan de Colima, Mexico: surface hydro-repellency as initiation process: Journal of Volcanology and Geothermal Research, 189, 105-117.

Dávila, N., Capra, L., Gavilanes-Ruiz, J.C., Varley, N., Norini, G., GomezVazquez, A., 2007, Recent lahars at Volcán de Colima (Mexico): drainage variation and spectral classification: Journal of Volcanology and Geothermal Research 165, 127-141.

Fernández-Lavado, C., 2010, Caracterización de la Inundabilidad en el Área Metropolitana de San Salvador: San Salvador, El Salvador, Geólogos del Mundo, programa IPGARAMSS, $144 \mathrm{pp}$.

FLO-2D Software INC., 2017, FLO-2D Reference Manual: Nutrioso, Estados Unidos, flo-2d.com, base de datos, <http://www.flo-2d.com/>, acceso libre, consulta: 09 de enero de 2017.

Gurdiel-Pérez, I., 2014, Aplicación del método geomorfológico histórico en la elaboración de cartografía de inundabilidad de Piña de Esgueva: Valladolid, España, Universidad de Valladolid, Trabajo de Fin de Grado, $67 \mathrm{pp}$.

Hernández, M., Núñez-Álvarez, L., Girón, L.I., Gutiérrez-López, R., 2012, Estudio Hidrológico-Hidráulico de avenida en el río San Francisco (Panajachel): Panajachel, Guatemala, Geólogos del Mundo - Vivamos Mejor - AECID, 83 pp.

Hsu, S.M., Chiou, L.B., Lin, G.F., Chao C.H., Wen, H.Y., Ku, C.Y., 2010, App licationsofsimulationtechniqueondebris-flowhazardzonedelineation: a case study in Hualien County, Taiwan: Natural Hazards Earth System Sciences, 10, 535-545.

Hürlimann, M., Copons, R., Altimir, J., 2006, Detailed debris flow hazard assessment in Andorra: A multidisciplinary approach: Geomorphology 78 (2006), 359-372, doi.org/10.1016/j.geomorph.2006.02.003.

INE (Instituto Nacional de Estadística de Guatemala), 2015, Base de datos: Ciudad de Guatemala, Guatemala, <https://www.ine.gob.gt/>, consulta 5 de febrero de 2015.

INSIVUMEH (Instituto Nacional de Sismología, Vulcanología, Meteorología e Hidrología), 2013, Base de datos, Registro de Datos Meteorológicos: Departamento de Meteorología, Ciudad de Guatemala, Guatemala, $<$ http://www.insivumeh.gob.gt/>, consulta 3 de abril de 2013.

Insuasty-Enríquez, J.E., 2012, Dinámica de flujos de lodos: Revista Unimar, 60, Julio-Diciembre, 2012, 43-54.

Julien, P.Y., Lan, Y., 1991, Rheology of hiper concentrations: Journal of Hydraulic Engineering, 117, 346-353.

Lin, P-S., Lee, J-H., Chang, C-W., 2011, An application of the FLO-2D model to debris-flow simulation - a case study of song-her district in Taiwan $E N 5^{\text {th }}$ International Conference on Debris-Flow Hazards "Mitigation, Mechanics, Prediction and Assessment", Topic 8 - Prediction and Assessment of Debris Flow Hazards: Italian Journal of Engineering Geology and EnvironmentBook, 947-956, DOI: 10.4408/IJEGE.2011-03.B-103.

López-Maldonado, C.E., 2014, Anteproyecto de diseño de sistema de abastecimiento de agua potable para la aldea Tzununá del municipio de Santa Cruz la Laguna, Departamento de Sololá: Ciudad de Guatemala,
Guatemala, Universidad Rafael Landívar, Tesis de Grado Licenciatura, $123 \mathrm{pp}$.

MAGA (Ministerio de Agricultura, Ganadería y Alimentación de Guatemala), 2010, Mapa digital de Usos del Suelo de Guatemala: 1:50,000, 1 mapa Ciudad de Guatemala, Guatemala, <https://www.maga.gob.gt/>, consulta: 6 de junio de 2013.

Montoya-Jaramillo, L.J., Silva-Arroyave, S.M., González, J.E., 2009, Evaluación de zonas de amenaza por avenidas torrenciales utilizando metodologías cualitativas. Caso de aplicación a la quebrada de Doña María: Revista Ingenierías Universidad de Medellín, 8(15), 11-29.

National Hurricane Center (NHC), 2018, Archive of Hurricane Seasons: Estados Unidos. National Oceanic and Atmospheric Administration (NOAA), National Hurricane Center (NHC), base de datos, <http://www.nhc.noaa. gov/>, acceso libre, consulta: 2 de febrero de 2016.

Newhall, C.G., 1987, Geology of the lake Atitlán region, western of Guatemala: Journal of Volcanology and Geothermal research, 33, 23-55.

Núñez-Álvarez, L., Martínez-Cobo, V., 2011a, Informe complementario de la cartografía geológica y geomorfológica (1:25.000) de la cuenca del lago Atitlán (Guatemala): Lago Atitlán, Guatemala, Geólogos del Mundo Vivamos Mejor - AECID, 69 pp.

Núñez-Álvarez, L., Martínez-Cobo, V., 2011b, Mapa Geológico de Guatemala 1:25.000 (Cuenca Atitlán): Lago Atitlán, Guatemala, Geólogos del Mundo - Vivamos Mejor - AECID, 1 mapa.

O'Brian, J.S., Julien, P.Y., Fullerton, W.T., 1993, Two-Dimensional Water Flood and Mudflow Simulation: Journal of Hydraulic Engineering, 119(2), 244-261.

Oliva-Hernández, J.P., 2007, Evaluación de amenaza por flujos de detritos en los poblados de Panabaj y Tzanchaj, Santiago de Atitlán, Guatemala Lago Atitlán, Guatemala, Universidad Nacional Autónoma de Nicaragua, Maestría Centroamericana en evaluación de Riesgo y Reducción de Desastres, $107 \mathrm{pp}$.

Paricio-Salas, S., 2011, Análisis geomorfológico de la susceptibilidad al alcance por corrientes de derrubios y avenidas torrenciales en la zona sur del lago Atitlán (Guatemala): Lago Atitlán, guatemala, Universitat de Barcelona (UB), Universitat Autònoma de Barcelona (UAB), Trabajo de Fin de Máster, 63 pp.

Pasuto, A., Silvano, S., 1998, Rainfall as a trigger of shallow mass movements. A case of study in the Dolomites, Italy: Environmental Geology, 35(2/3), 184-189.

Pierson, T.C., 2005, Distinguishing between Debris Flows and Flood sfrom Field Evidence in Small Watersheds: USGS Fact Sheet, 2004-3142.

Pierson, T.C., Costa, J.E., 1987, A rheologic classification of subaerial sedimentwater flows: Geological Society of America, Reviews in Engineering Geology, 7, 1-12.

Sepúlveda, A., Patio, J., Rodríguez, C.E., 2016, Metodología para evaluación de riesgo por flujo de detritos detonados por lluvia: caso Útica, Cundinamarca, Colombia: Obras y Proyectos 20, 31-43.

Serra-Creixell, G., 2011, Estudio de inundabilidad en la zona norte del lago Atitlán (Guatemala): Lago Atitlán, Guatemala, Universitat de Barcelona (UB), Universitat Autònoma de Barcelona (UAB), Trabajo de Fin de Máster, 31 pp.

Stevens, N.F., Manville, V., Heron, D.W., 2002, The sensitivity of a volcanic flow model to digital elevation model accuracy: experiments with digitized map contours and interferometric SAR at Ruapehu and Taranaki volcanoes, New Zealand: Journal of Volcanology and Geothermal Research, 119, 89-105.

USACE (US Army Corps of Engineers), 2018, Hydrologic Engineering Center (HEC), < https://www.hec.usace.army.mil//>, acceso libre, consulta 21 de septiembre de 2015.

VanDine, D.F., 1996, Debris flow control structures for forest engineering: Victoria, Columbia Británica, Canadá, Ministry of Forests, Research Program, 75 pp.

Vilaplana, J.M., Pallás, R., Guinau, M., Falgás, E., Alemany, X., 2002, Propuesta para una cartografía de peligrosidad geológica asociada a los movimientos de ladera en el noroeste de Nicaragua: Serie Geográfica, 10, 109-119.

Manuscrito recibido: abril 2, 2018

Manuscrito corregido recibido: octubre 14, 2018

Manuscrito aceptado: noviembre 6, 2018 